\title{
New Technologies for Nanobiosensing and their Applications to Real-Time Monitoring
}

\author{
Cristina Boero, Sandro Carrara and Giovanni De Micheli \\ EPFL - École Politechnique Fédérale de Lausanne \\ Lausanne, 1015 - Switzerland \\ Email: cristina.boero@epfl.ch
}

\begin{abstract}
Amperometric biosensors are complex systems and they require a combination of technologies for their development. The aim of the present work is to exploit existing technologies for new purposes in order to develop nanostructured biosensors for the real-time detection of multiple metabolites in cell culture flasks. The fabrication of five Au working electrodes onto silicon substrate is achieved with CMOS compatible microtechnology. Each working electrode presents an area of $0.25 \mathrm{~mm}^{2}$, so structuration with carbon nanotubes and specific functionalization are carried out by using spotting technology, originally developed for microarrays and DNA printing. The electrodes are characterized by cyclic voltammetry and compared with commercially available screen-printed electrodes. Finally, they are functionalized with different enzymes and calibrated for real-time detection of glutamate, glucose and lactate under flow conditions. The developed biosensor for real-time and online detection of multiple metabolites shows very promising results towards its application in cell culture monitoring.
\end{abstract}

\section{INTRODUCTION}

Electrochemical biosensors are complex systems and consist of heterogeneous components: the electrochemical cell, typically with three electrodes; the biological recognition element; the electronics for carrying out measurements; the fluidics, especially for real-time detection. Different technologies are required to fabricate and assemble all these components together, and sometimes conventional techniques are not suitable for this purpose, especially in the case of miniaturized systems. The microfabrication of electrodes can be performed with CMOS compatible technologies, which allow us to integrate in the same chip both electrodes and electronics, and shrink everything down to the micro-scale. Electrodes can be made of gold, platinum, or silver by evaporation onto silicon substrate. Afterwards, the sensing element has to be placed on the working electrode. The sensing element for biosensors is typically a protein, which reacts with the target molecule. The straightforward way to deposit protein on the electrodes is by drop casting with pipettes. However, the technique is no longer suitable when electrodes are miniaturized. Thus, we borrow the spotting technology from the field of microarrays and DNA printing. Spotting tools allow us to handle ultralow volume liquid and precisely place drops of $50 \mu \mathrm{m}$ in diameter. Spotting can help us also if we want to modify the electrode surface, for examples by placing nanomaterials. Carbon nanotubes (CNT) have shown really good properties for biosensing [1] and can be used to this purpose. They have to be in solution and well dispersed for the spotting, in a non-volatile solvent, since the spotted volume can be down to $500 \mathrm{pl}$. Real-time monitoring requires fresh solution at the electrode surface and equal concentration between the bulk and the interface and for these reasons the fluidic system plays a central role. Hence, it is also necessary to integrate a fluidic cell next to the electrodes to create a miniaturized electrochemical cell. Channels and chamber can be manufactured in different materials: to this purpose the most used in literature is PDMS (polydimethylsiloxane), which is biocompatible and presents several other advantages, like polymerization at room temperature [2] and the possibility to be attached reversibly or irreversibly onto silicon-based materials by oxygen plasma [3]. However, it is also possible to use more rigid materials, like methacrylate combined with o-rings to ensure hermetic enclosure, as we use in the present research.

All the aforementioned steps need to be combined together. The aim of the present paper is to propose existing technologies for the development of fully integrated biosensors for cell monitoring. The final goal of our research is to achieve the detection of multiple metabolites with a unique platform integrating electrodes, electronics and fluidics for the online and real-time detection of such compounds in cell culture medium.

\section{Metabolite Detection in CELl CUlture Medium}

There is an increasing interest about metabolite levels in cell cultures, as their monitoring can reveal unknown mechanisms and be a further tool for cell analysis. Among all the endogenous compounds involved in cell cycle, glucose and lactate play an important role. Glucose is the main source of energy for cells and when it runs out in the medium, biologists need to replace it. Lactate, instead, is a marker of cell sufferance, for lack of feeding or oxygen. In medical practise lactate is often monitored when some organs have risk of ischemia. In our previous work [4], we have already shown that it is possible to detect such metabolites off-line during different cell cycles. When dealing with neural cell lines, another important compound to take into account is glutamate, the most important excitatory neurotransmitter in the mammalian central nervous system. Its monitoring can be fundamental for the investigation of neurological disorders [5]. There are other several interesting compounds to be monitored in cell cultures, like ethanol, 1-amino acids, galactose, etc [6]. To date, the majority of research on this topic is focused on the 


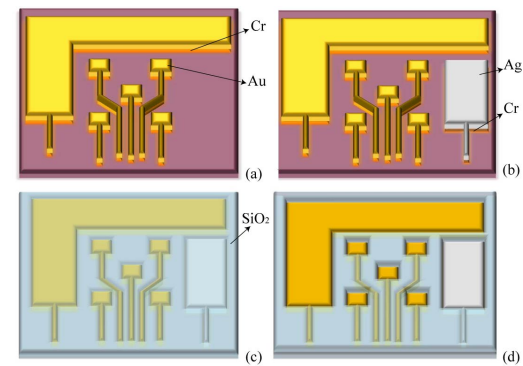

Fig. 1. Process flow for fabrication of the electrochemical cell with multiple WEs: (a) $\mathrm{Cr} / \mathrm{Au}$ deposition; (b) $\mathrm{Cr} / \mathrm{Ag}$ deposition; (c) $\mathrm{SiO}_{2}$ sputtering; (d) $\mathrm{SiO}_{2}$ wet etching.

development of integrated chips where cells can be inoculated and cultivated [7], [8]. On the other hand, minimizing interferences with cells is much more desirable. CITSens Bio sensors (C-CIT, Switzerland) are the only example in the market able to perform real-time cell monitoring, even if they do not allow multiple detection on the same platform.

In the present research we use a particular type of protein, the oxidase, as sensing element, which generates hydrogen peroxide $\left(\mathrm{H}_{2} \mathrm{O}_{2}\right)$ when reacting with the target molecule. The use of this particular recognition element enables us to achieve the monitoring of different targets with the same detection principles. In fact, once the oxidase reacts with its specific substrate, hydrogen peroxide is produced as reaction product. The electrical detection occurs because $\mathrm{H}_{2} \mathrm{O}_{2}$ is an electrochemical active species which oxidates when a DC potential of +650 $\mathrm{mV}$ is applied. Therefore, the current intensity is proportional to the metabolite concentration reacting with the enzyme. Such measurements are typically done by a potentiostat instrument. Previous work [9] showed how to integrate the potentiostat function in an IC. The main steps for biosensor fabrication are considered in the following sections.

\section{MICROFABRICATION AND FUNCTIONALIZATION}

The detection of multiple targets requires multiple working electrodes (WEs). We nanostructure the WEs by using carbon nanotubes (CNT), since they have specific electronic properties, like good conductivity [10], high electron transfer rate [6], and considerable electronic emission [11]. These properties result in enhanced sensitivities and lower detection limit from the point-of-view of the biosensor [12], [?]. Then, different protein functionalization of WEs will guarantee the multiple detection of metabolites.

\section{A. Microfabrication}

The chip is conceived with five working electrodes, in order to detect up to five different metabolites at the same time. It consists of gold working electrodes, which will be nanostructured afterwards, gold counter electrode, and silver reference electrode. A schematic of the microfabrication process is illustrated in Fig. 1. Metal layers are evaporated by physical vapor deposition (PVD) onto $525 \mu \mathrm{m}<100>$ silicon substrate covered by $500 \mathrm{~nm}$ of $\mathrm{SiO}_{2}$. $\mathrm{Au}$ and $\mathrm{Ag}$ thin films $(200 \mathrm{~nm})$ are patterned to form the electrodes and connections

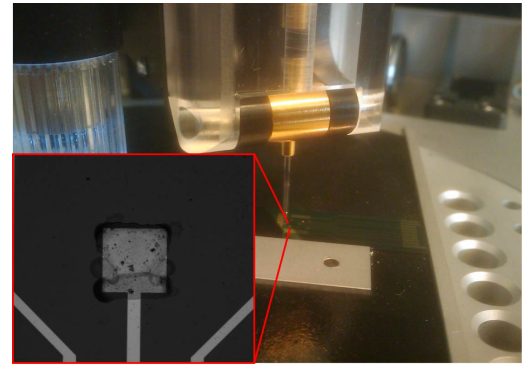

Fig. 2. Spotting system for CNT deposition. Inset: optical image of one working electrode covered with CNT dissolved in Nafion solution (Microscope Nikon Eclipse LV100).

by a lift-off process. A thin film of $\mathrm{Cr}(20 \mathrm{~nm})$ is used as stick layer for both the metals. All the WEs are identical and they have an area of $0.25 \mathrm{~mm}^{2}$. Connector insulation is achieved by radio-frequency (RF) sputtering of $\mathrm{SiO}_{2}$ and successive wet etching with hydrofluoric acid (BHF 7:1) next to the electrodes and pads for electronic connections. The interface between the chip and the potentiostat is guaranteed by using a commercial edge card socket, while the switching among the five WEs is achieved by a manual switch in this first prototype.

\section{B. CNT spotting}

Multi-walled carbon nanotubes (MWCNT - diameter 10 $\mathrm{nm}$, length 1-2 $\mu \mathrm{m}$ ) are purchased in powder (90\% purity) from Dropsens (Spain). Then, they are dispersed in a solution of distilled water $50 \mathrm{vv} \%$ and ethanol $50 \mathrm{vv} \%$, with $0.5 \mathrm{wt} \%$ of Nafion (purchased from Sigma-Aldrich, Switzerland). Final concentration of MWCNT is $1 \mathrm{mg} / \mathrm{ml}$. The solution based on Nafion is chosen according to the really good results reported from Wang et al. [13] and it is preferred to dispersion in chloroform, which is too volatile for the volumes involved in our research. Automatic spotting of CNT is performed with a commercially available non-contact spotter (sciFLEXARRAYER DW by Scienion, Germany). The minimum dispensed volume is around $400 \mathrm{pl}$ and can be increased by increasing the number of drops per spot. According to previous results obtained with macroelectrodes [14], we deposit $600 \mathrm{ng}$ of CNT, corresponding to a volume of $600 \mathrm{nl}$ of MWCNT/Nafion solution. Since the drops have a diameter around 50-80 $\mu \mathrm{m}$ and they tend to aggregate (the walls of carbon nanotubes are highly hydrophobic), we pattern the electrode area as a matrix of $5 \times 5$ spots, to cover all the exposed area. Then, we dispense 10 drops for each spot (around $4 \mathrm{nl}$ per spot) for 5 cycles. The dimension and volume of the drops can be monitored at the end of each spotting by using a camera installed on the instrument. The setup and the result of the spotting are illustrated in Fig. 2. Nafion solution is allowed to dry for every spotting and carbon nanotubes perfectly cover the electrode surface.

\section{Functionalization with enzymatic probes}

In the present research we want to show results related to glucose, lactate, and glutamate detection, but the same principles can be used for other metabolite detections. Glucose 


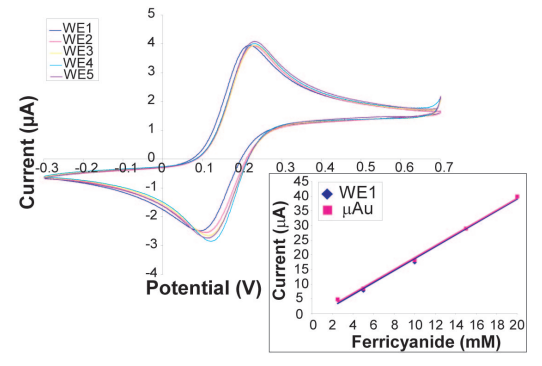

Fig. 3. Cyclic voltammograms of $10 \mathrm{mM}$ ferricyanide for the five working electrodes. Inset: comparison between commercially SPE and microfabricated electrodes for ferricyanide.

oxidase from Aspergillus Niger (GOD, EC 1.1.3.4, 129.9 units/mg solid), glutaraldehyde solution (25\% in water), D-(+)glucose, lithium L-lactate, and L-glutamic acid were purchased from Sigma-Aldrich (Switzerland) as lyophilized powders. Glutamate oxidase from Streptomyces species (GlOD, EC 1.4.3.11, 25 units) was supplied from Yamasa Co. (Japan), while lactate oxidase from Pediococcus species (LOD, EC 1.13.12.4, $\geq 20$ units/mg solid) was purchased from Roche Diagnostics GmbH (Germany). All the enzymes are dissolved in Phosphate Buffer Saline solution (PBS) 0.01 M at pH 7.4 and glutaraldehyde $0.25 \%$ (GOD $50 \mathrm{mg} / \mathrm{ml}$, LOD $66 \mathrm{mg} / \mathrm{ml}$, and GIOD $0.6 \mathrm{U} / \mu \mathrm{l}$ ), while metabolites are dissolved only in PBS solution. For GOD and LOD the enzyme concentration is enough to spot 100 drops for each electrode $(5 \times 5$ matrix, 4 drops per spot), while GIOD is more diluted, so we need 1000 drops onto the electrode area $(10$ x 10 matrix, 10 drops per spot).

\section{Measurement Results}

Due to the accuracy of lithographic process, first we need to characterize the electrodes alone to see how the electrical properties vary, especially in terms of active area. To this purpose we exploit them with cyclic voltammetry (CV), using ferricyanide, which is a well-known and stable species. Afterwards, we compare the recorded CV with those obtained with commercially available screen-printed electrodes (SPE) (Dropsens, Spain). Finally, once the electrodes are nanostructured and functionalized, we proceed with their calibration according to the enzyme probe. All the electrochemical measurements are acquired by using Autolab potentiostat system (Metrohm, Netherlands).

\section{A. Electrode characterization}

Ferricyanide solutions are prepared in the range of $0-20$ $\mathrm{mM}$ in PBS (ferricyanide is purchased in powder from SigmaAldrich, Switzerland). For CV characterization, electrodes are kept in quiescent conditions, by casting a $100 \mu \mathrm{l}$ drop of substrate to cover the whole electrochemical cell. We perform 10 cycles and we consider the last one, which is the most stable. We repeat the measurements for several concentrations of ferricyanide for all the five electrodes. Fig. 3 illustrates an example of cyclic voltammetry performed on the five WE for $10 \mathrm{mM}$ of ferricyanide. The CVs overlap for different

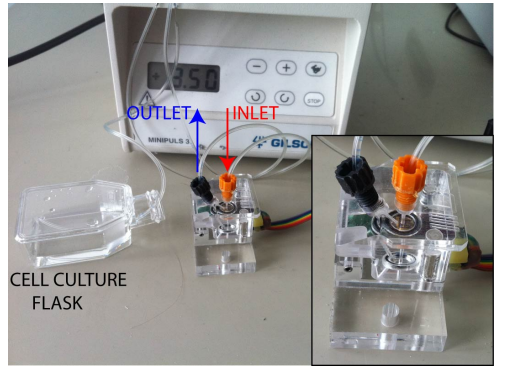

Fig. 4. Fluidic system for calibration of different metabolites. Inset: the fluidic cell hosting multi-working electrode chip.

electrodes and the two peaks of ferricyanide are well shaped. The average potential for the oxidation peaks is $229.4 \pm 7.3$ $\mathrm{mV}$, while is $109.2 \pm 8.7 \mathrm{mV}$ for reduction peaks. Therefore, the difference among different electrodes is lower than $10 \%$ (much lower in the case of oxidation peak, which is the one we use for the following measurements).

Peak height varies according to substrate concentration under cyclic voltammetry for both SPE and microfabricated electrodes. If we compare the two calibration lines, once they are normalized for the electrode area, we notice an almost perfect superimposition, as shown in the inset of Fig. 3. That result guarantees that microfabricated electrodes are suitable to be used as electrochemical biosensors.

\section{B. Calibration for different metabolites}

Calibrations for the different metabolites are performed by means of chronoamperometry under flow conditions with nanostructured and functionalized electrodes. The microfabricated chip is inserted in a flow-cell (Dropsens, Spain), which is connected to a peristaltic pump (Gilson SA, Switzerland) by means of Tygon tubes of $0.25 \mathrm{~mm}$ internal diameter. The experimental setup is shown in Fig. 4. The sample solution is sucked from the cell culture flask with a velocity of around 13 $\mu \mathrm{l} / \mathrm{min}$. Once the measurement is run, we change the solution at the inlet of the fluidic circuit around every 3 minutes. As result of solution changing, we have current variation on the active working electrode. A typical chronoamperometry is depicted in Fig. 5: each step corresponds to a different concentration of glutamate. In this case the system is not responding for concentration lower than $400 \mu \mathrm{M}$, while, afterwards, we have well-shaped steps every time the substrate is injected. Then, the biosensor reaches saturation above $1 \mathrm{mM}$. Calibration line is calculated from current steps, by measuring the difference between the baseline (related to $0 \mathrm{mM}$ concentration) and the reached value. For glutamate the sensitivity results in a value of $0.42 \mathrm{nA} / \mu \mathrm{M} \mathrm{cm}^{-2}$ and a detection limit of $216 \mu \mathrm{M}(\mathrm{S} / \mathrm{N}=$ $3 \sigma)$. We perform chronoamperometries for glucose and lactate with the same setup used for glutamate. The sensitivity for glucose is $0.46 \mathrm{nA} / \mu \mathrm{M} \mathrm{cm}{ }^{-2}$ with a detection limit of 115 $\mu \mathrm{M}$, while for lactate is $1.09 \mathrm{nA} / \mu \mathrm{M} \mathrm{cm}^{-2}$ with a detection limit of $115 \mu \mathrm{M}$, as well. Calibrations for the three metabolites are illustrated in Fig. 6.

Those results show that our sensors are suitable for real-time 


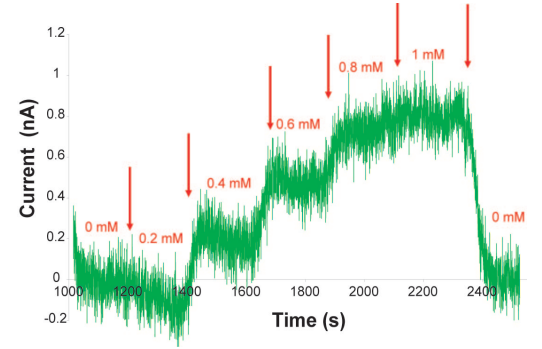

Fig. 5. Typical chronoamperometry for glutamate in the range within 0 and $1 \mathrm{mM}$.

and online monitoring of metabolites in cell culture media. The fluidic system allows media dilution for measuring purposes [?], [14], and such dilutions fit very well with concentration windows measured with our biochip.

\section{Conclusions}

We described how various heterogeneous technologies can support the development of electrochemical biosensors for the detection of multiple metabolites, such as glucose, lactate and glutamate. We have fabricated a micro-electrochemical cell with five working electrodes. We have shown that it is possible to nanostructure and functionalize WEs by using spotting tools, which is mainly developed for microarrays and DNA printing. We have demonstrated that redox reactions on microfabricated electrodes are the same as those obtained with commercially available gold screen-printed electrodes. We achieved the selective functionalization of the electrodes with different oxidases and we calibrated under flow conditions the developed biosensor for glucose, lactate and glutamate detection. We obtained a sensitivity of $0.42 \mathrm{nA} / \mu \mathrm{M} \mathrm{cm}^{-2}$ and a detection limit of $216 \mu \mathrm{M}$ for glutamate, a sensitivity of 0.46 $\mathrm{nA} / \mu \mathrm{M} \mathrm{cm}^{-2}$ and a detection limit of $115 \mu \mathrm{M}$ for glucose, a sensitivity of $1.09 \mathrm{nA} / \mu \mathrm{M} \mathrm{cm} \mathrm{cm}^{-2}$ and a detection limit of $115 \mu \mathrm{M}$ for lactate. Few works [15], [16] deal with multiple detection of metabolites in cell cultures and none of them has shown the integration of multiple and nanostructured WEs sharing common counter and reference electrode. Instead, the results presented in this work represent a novelty with respect to the state-of-the-art in cell culture monitoring and pave the way to new integrated systems for cell analysis.

\section{ACKNOWLEDGMENT}

The authors would like to thank all the CMI staff for their support on microfabrication processes. Financial supports are from the Swiss Science National Foundation (project "Nanostructured biochip development for stem cell monitoring" grant $n$. IZLCZ2 123967 by Sino-Swiss cooperation) and from NanoSys project (program ERC-2009-AdG-246810).

\section{REFERENCES}

[1] Sandro Carrara, Cristina Boero, and Giovanni Micheli. Quantum dots and wires to improve enzymes-based electrochemical bio-sensing. In Nano-Net, volume 20, pages 189-199. Springer Berlin Heidelberg, 2009.

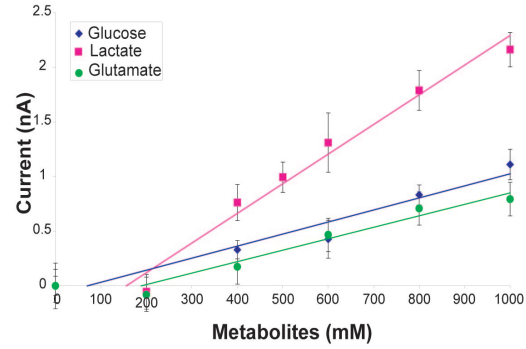

Fig. 6. Calibration lines for the three considered metabolites: glucose (blue rhombus), lactate (pink square), and glutamate (green dots).

[2] J. C. McDonald and G. M. Whitesides. Poly(dimethylsiloxane) as a material for fabricating microfluidic devices. Accounts of Chemical Research, 35(7):491-499, 2002.

[3] K. Chau, B. Millare, A. Lin, S. Upadhyayula, V. Nuñez, H. Xu, and V. Vullev. Dependence of the quality of adhesion between poly(dimethylsiloxane) and glass surfaces on the composition of the oxidizing plasma. Microfluidics and Nanofluidics, 10:907-917, 2011.

[4] Cristina Boero, Sandro Carrara, Giovanna Del Vecchio, Laura Calz, and Giovanni De Micheli. Targeting of multiple metabolites in neural cells monitored by using protein-based carbon nanotubes. Sensors and Actuators B: Chemical, 157(1):216 - 224, 2011.

[5] M. A. Rahman, N. Kwon, M. Won, E. S. Choe, and Y. Shim. Functionalized conducting polymer as an enzyme-immobilizing substrate: an amperometric glutamate microbiosensor for in vivo measurements. Analytical Chemistry, 77(15):4854-4860, 2005.

[6] V. Vojinovic, F.M.F. Esteves, J.M.S. Cabral, and L.P. Fonseca. Bienzymatic analytical microreactors for glucose, lactate, ethanol, galactose and 1-amino acid monitoring in cell culture media. Analytica Chimica Acta, 565(2):240 - 249, 2006.

[7] J. Wiest, M. Schmidhuber, J. Ressler, A. Scholz, M. Brischwein, and B. Wolf. Cell based assays for diagnostic and therapy on multiparametric biosensor chips with an intelligent mobile lab. In IFMBE Proceedings, volume 10, pages 132-135, 2005.

[8] N. Pereira Rodrigues, Y. Sakai, and T. Fujii. Cell-based microfluidic biochip for the electrochemical real-time monitoring of glucose and oxygen. Sensors and Actuators B: Chemical, 132(2):608 - 613, 2008.

[9] C. Boero, S. Carrara, and G. De Micheli. Sensitivity enhancement by carbon nanotubes: Applications to stem cell cultures monitoring. In Research in Microelectronics and Electronics, 2009. PRIME 2009. Ph.D., pages $72-75$, july 2009.

[10] K. Gong, Y. Yan, M. Zhang, L. Su, S. Xiong, and L. Mao. Electrochemistry and electroanalytical applications of carbon nanotubes: a review. Analytical science, 21:1383 - 1393, 2005.

[11] A. Mayer, N. M. Miskovsky, and P. H. Cutler. Theoretical comparison between field emission from single-wall and multi-wall carbon nanotubes. Physical Review B, 65(15):155420, 2002.

[12] S. Carrara, V.V. Shumyantseva, A.I. Archakov, and B. Samorì. Screenprinted electrodes based on carbon nanotubes and cytochrome p450scc for highly sensitive cholesterol biosensors. Biosensensors and Bioelectronics, 24(1): 148 - 150, 2008.

[13] J. Wang, M. Musameh, and Y. Lin. Solubilization of carbon nanotubes by nafion toward the preparation of amperometric biosensors. Journal of the American Chemical Society, 125(9):2408-2409, 2003.

[14] C. Boero, S. Carrara, G. Del Vecchio, L. Calzá and, and G. De Micheli. Highly sensitive carbon nanotube-based sensing for lactate and glucose monitoring in cell culture. IEEE Transactions on NanoBioscience, 10(1):59-67, 2011.

[15] J. Perdomo, H. Hinkers, C. Sundermeier, W. Seifert, O. Martnez Morell, and M. Knoll. Miniaturized real-time monitoring system for -lactate and glucose using microfabricated multi-enzyme sensors. Biosensors and Bioelectronics, 15(9-10):515 - 522, 2000.

[16] M. Odijk, A. Baumann, W. Lohmann, F.T.G. van den Brink, W. Olthuis, U. Karst, and A. van den Berg. A microfluidic chip for electrochemical conversions in drug metabolism studies. Lab on a Chip, 9:1687-1693, 2009. 